\title{
Cellular allorecognition and its roles in Dictyostelium development and social evolution
}

\author{
PETER KUNDERT ${ }^{1,2}$ and GAD SHAULSKY*,1 \\ ${ }^{1}$ Department of Molecular and Human Genetics, Baylor College of Medicine, Houston, TX, USA and \\ ${ }^{2}$ Medical Scientist Training Program, Baylor College of Medicine, Houston, TX, USA
}

\begin{abstract}
The social amoeba Dictyostelium discoideum is a tractable model organism to study cellular allorecognition, which is the ability of a cell to distinguish itself and its genetically similar relatives from more distantly related organisms. Cellular allorecognition is ubiquitous across the tree of life and affects many biological processes. Depending on the biological context, these versatile systems operate both within and between individual organisms, and both promote and constrain functional heterogeneity. Some of the most notable allorecognition systems mediate neural self-avoidance in flies and adaptive immunity in vertebrates. $D$. discoideum's allorecognition system shares several structures and functions with other allorecognition systems. Structurally, its key regulators reside at a single genomic locus that encodes two highly polymorphic proteins, a transmembrane ligand called TgrC1 and its receptorTgrB1. These proteins exhibit isoform-specific, heterophilic binding across cells. Functionally, this interaction determines the extent to which co-developing $D$. discoideum strains co-aggregate or segregate during the aggregation phase of multicellular development. The allorecognition system thus affects both development and social evolution, as available evidence suggests that the threat of developmental cheating represents a primary selective force acting on it. Other significant characteristics that may inform the study of allorecognition in general include that $\boldsymbol{D}$. discoideum's allorecognition system is a continuous and inclusive trait, it is pleiotropic, and it is temporally regulated.
\end{abstract}

KEY WORDS: Dictyostelium, allorecognition, $\operatorname{tgr} B 1, \operatorname{tgr} C 1$, development, social evolution

\section{Allorecognition is an important feature of biology}

Cellular allorecognition, the ability of a cell to distinguish itself and its genetically similar relatives from more distantly related organisms, is a common feature of cellular life. Allorecognition is found in diverse cellular contexts: it is present in prokaryotes and eukaryotes and it abounds in unicellular as well as obligately and facultatively multicellular organisms. While the focus of this review is Dictyostelium allorecognition, we will briefly explore the phenomenology of allorecognition in each of these contexts to demonstrate its frequency and general significance in biology. We will broadly categorize these allorecognition systems as occurring between (inter-) versus within (intra-) individual organisms, whether unicellular or multicellular. We will also note whether the allorecognition systems promote functional heterogeneity or constrain it. This last classification refers to the proposed functions of the allorecognition systems. As defined here, heterogeneity is distinct from the mechanisms that promote the requisite diversity - genetic or otherwise-to distinguish between self and non-self that are a necessary feature of all allorecognition systems. For example, an allorecognition system that promotes functional heterogeneity might physically bring together genetically heterogeneous individuals or groups as a means to accomplish a biological process, while a system that constrains functional heterogeneity might prevent individuals or groups from interacting during an analogous process.

\section{Allorecognition in prokaryotes}

Individual unicellular bacteria widely exhibit intra-individual allorecognition that promotes functional heterogeneity in their immune defenses against bacteriophages, both innately with restriction enzymes and adaptively with CRISPR/Cas systems (Seed 2015;

Abbreviations used in this paper: E-set, early set; Gp150, 150 kD glycoprotein; IG, immunoglobulin; IPT, Ig-like, plexins, transcription factor; lag (gene prefix), loose aggregate arrest; $\operatorname{tgr}$ (gene prefix), transmembrane.

\footnotetext{
*Address correspondence to: Gad Shaulsky. Department of Molecular and Human Genetics, Baylor College of Medicine, Houston, TX 77030, USA. Tel: +1-713 798 8082. E-mail: gadi@ bcm.edu - (ID) https://orcid.org/0000-0002-0532-0551
} 
Hille et al., 2018). Many unicellular bacteria also distinguish between themselves at intra- and inter-species levels in both competitive and cooperative interactions (Strassman et al., 2011).

An example of inter-individual allorecognition that constrains functional heterogeneity in a prokaryote occurs in Proteus mirabilis, which causes kidney stones in humans. These bacteria rely on cellcell contact and cooperative motility to mediate rapid movement across surfaces in a behavior called swarming. When isogenic $P$. mirabilis colonies meet, they fuse and swarm together; whereas when non-isogenic colonies meet, they form visible boundaries. The mechanism that underlies this selective cooperative motility involves a pair of polymorphic genes of the ids family that are tightly linked on the bacterial chromosome. The analogous locus in $D$. discoideum called tgr is similarly a pair of tightly linked, highly polymorphic genes. The protein products of the ids genes are transferred between bacteria, and cause swarming only in compatible cells (Cardarelli et al., 2015; Saak and Gibbs 2016).

The facultatively multicellular gram-negative prokaryote Myxococcus xanthus exhibits inter-individual allorecognition that constrains functional heterogeneity in a developmental response to starvation, similar to that of Dictyostelium amoebae. These similarities include the formation of terminal fruiting bodies, cell differentiation into spores and auxiliary cell types, the natural occurrence of obligate cheaters that disproportionately contribute to spores when codeveloped with other strains, and the use of allorecognition to influence whether strains fruit together or separately. A function of the allorecognition system in $M$. xanthus that is distinct from allorecognition in Dictyostelium is to maintain separation between growing colonies of different allotypes that may contact each other during vegetative growth. This phenomenon is called colony-merger incompatibility. One potential regulator of $M$. xanthus allorecognition is traA. This gene encodes an outer membrane protein that mediates outer membrane exchange (OME), a process in which $M$. xanthus bacteria exchange outer membrane lipids and associated proteins. TraA has a hypervariable domain that confers a unique allotype to each $M$. xanthus strain, and OME occurs only between strains with matching allotypes. While the TraA allotypes of mixed strains do influence coordinated motility and immunity from interstrain killing (Pathak et al., 2013), they do not predict colony-merger incompatibilities (Wielgoss et al., 2018). This observation suggests that additional signaling mechanisms exist to mediate $M$. xanthus allorecognition (Bretl and Kirby 2016).

\section{Allorecognition in eukaryotes}

One example of inter-individual allorecognition that constrains functional heterogeneity in a unicellular eukaryote is the process of flocculation in the yeast Saccharomyces cerevisiae. Flocculation is a cell-aggregation process that protects against environmental insults such as antimicrobials. The gene that mediates this form of cell-cell adhesion is called flo1. A given yeast cell can effectively adhere to other flocculating cells only if it expresses FLO1. This selective adherence prevents cells that do not express flo 1 from receiving the protective benefit of grouping together. flo1 thus represents an example of a green-beard gene, which is a gene that can recognize copies of itself in conspecifics and selectively confer benefit to them (Smukalla et al., 2008; Dawkins 1976). There have been two green-beard loci described in Dictyostelium. Queller et al. characterized a green-beard gene in $D$. discoideum called
csaA (Queller et al., 2003), however it is not polymorphic and there is no evidence to suggest that it is involved in allorecognition. In contrast, Gruenheit et al. recently described the tgr locus, a central regulator of $D$. discoideum allorecognition, as a polymorphic green-beard (Gruenheit et al., 2017). We will review their findings in depth later in this review.

Obligate multicellular eukaryotes have provided a bevy of examples of allorecognition systems that underlie an incredible diversity of biological processes. An example of an inter-individual allorecognition system that constrains functional heterogeneity in a multicellular eukaryote is found in the colonial ascidian Botryllus schlosseri. A founding organism of this sea squirt asexually buds to generate a colony that comprises additional genetically identical individuals that share an extracorporeal vascular system. Allorecognition with other colonies occurs when two colonies' extracorporeal vascular systems make contact. If the colonies are compatible, their vascular systems fuse; if they are incompatible, the interacting structures are destroyed and replaced with a scar. The proposed function of this allorecognition system is to protect individuals against germ cell parasitism, a process in which germ cells from one colony migrate through fused common vasculature into other colonies and compete with their germ cells for germline niches. A single genomic locus called fuhc (fusion/histocompatibility), which contains multiple genes implicated in allorecognition, is responsible for these outcomes. Specifically, colonies that share one or both fuhc haplotypes fuse, whereas those that share none reject (Taketa and De Tomaso 2014).

A second inter-individual allorecognition system that constrains functional heterogeneity in a multicellular eukaryote is found in the cnidarian Hydractinia symbiolongicarpus. Like B. schlosseri, when two colonies of this saltwater invertebrate make contact, they engage in a fusion/rejection interaction. Also like in B. schlosseri, the avoidance of germ cell parasitism may be the selective pressure that shapes $H$. symbiolongicarpus allorecognition. The genetic determinants of allorecognition in $\mathrm{H}$. symbiolongicarpus are two tightly linked and highly polymorphic genes called $A / r 1$ and Alr2 (Nicotra et al., 2009). These genes encode transmembrane proteins with multiple extracellular immunoglobin superfamily-like domains. ALR1 and ALR2 both exhibit isoform-specific, homophilic binding in trans (Karadge et al., 2015). This type of protein interaction is similar to that of the central regulators of allorecognition in D. discoideum, TgrB1 and TgrC1 (Nicotra et al., 2017; Hirose et al., 2017).

Vertebrate adaptive immunity is the best-studied example of allorecognition. In different contexts, this allorecognition systems operates both intra- and inter-individually, and to both promote and constrain functional heterogeneity. Within individuals, to generate the functional molecular heterogeneity required to recognize a vast amount of pathogen- and cancerous cell-derived peptides, the targeted and actively mutagenic process of $\mathrm{V}(\mathrm{D}) \mathrm{J}$ recombination occurs in the genes that encode $T$ cell receptors (TCRs) and B cell receptors (BCRs) during the early development of their respective cell types. In the case of TCRs, V(D)J recombination can generate about $3 \times 10^{11}$ unique receptors with binding specificities for distinct ligands. These ligands comprise peptides bound to transmembrane MHC proteins, which facilitate their presentation to TCRs. In this way, MHC-bound peptide ligands report the presence of extracellular pathogens as well as viral- and cancer-associated peptides within cells (Abbas 2015). Pathogen diversity has likely provided 
strong selection that has acted on the $\mathrm{MHC}$ locus and resulted in its high degree of polymorphism (Meyer et al., 2018). An unfortunate modern consequence of this allorecognition system is transplant rejection (Marino et al., 2016). However, rejection may be adaptive in that it can prevent transmission of cancerous cells between individuals. Such transmission is occurring between Tasmanian devils in the form of bites that transmit MHC-deficient oral cancer cells (Rock et al., 2019; Siddle et al., 2013). Cancerous cells and pathogens have correspondingly evolved many mechanisms to disrupt antigen processing and presentation and avoid immune recognition (Rock et al., 2019; Reeves and James 2016; Schuren et al., 2016). Medically, the field of cancer immunotherapy is founded on modulation of these pathways, and many of the advances in cell-based therapies are possible because of basic biological knowledge about the underlying mechanisms of this allorecognition system (Johnson and June 2017). We anticipate that insights from $D$. discoideum and other model organisms will inform future lines of inquiry to uncover common structures and functions in this clinically relevant allorecognition system.

Vertebrate adaptive immunity as an allorecognition system acts in two additional ways. First, it operates within individuals to constrain functional heterogeneity. In the case of T cells, the process of positive selection ensures that TCRs can effectively bind to $\mathrm{MHC}$, and the process of negative selection prevents autoimmunity by eliminating clones of T cells that bind to MHC-presented self-antigens (Abbas et al., 2015; Takaba and Takayanagi 2017). Second, it operates between individuals to promote heterogeneity by influencing mate choice. Researchers have clearly demonstrated this phenomenon in mice, but its relevance in modern human society remains controversial (Yamazaki and Beauchamp 2007). These additional functions of vertebrate adaptive immunity underscore that multiple evolutionary processes may often coopt allorecognition machinery.

Another example of multicellular eukaryotic allorecognition occurs in the processes of neuronal self-avoidance and circuit assembly. Neuronal self-avoidance is the process by which a given neuron's branches avoid synapsing on that same neuron's cell body, thus avoiding 'short circuits'. This process is necessary for proper neuronal wiring, likely because it ensures that dendritic territories are covered completely and non-redundantly (Hattori et al., 2007). This system operates within individuals and promotes functional heterogeneity. The locus that encodes the key components of this system in the fruit fly Drosophila melanogaster is called Dscam1. Through a series of mutually exclusive alternative splicing events at four exon clusters, this single locus can encode over 10,000 unique transmembrane protein isoforms that exhibit isoform-specific homophilic binding. Individual cells stochastically express several Dscam isoforms at a given time, and certain neuronal subtypes express particular groups of isotypes at higher rates than others (Neves et al., 2004). Dscam1-null mutants exhibit a complete lack of dendrite self-avoidance. Reintroduction of a single Dscam1 isoform can restore self-avoidance, but these neurons' dendrites cannot share the same territory, which results in defects in neural circuits (Hattori et al., 2007). As the potential number of isoforms increases, so too does the functionality of the neural circuits (Hattori et al., 2009). An analogous allorecognition system featuring similarly homophilic transmembrane proteins called atypical cadherins and clustered protocadherins exists in vertebrates to mediate neuronal self-avoidance and other aspects of neural patterning. The mecha- nism of generating isotype diversity in clustered protocadherins differs from the mutually exclusive alternative splicing of Dscam1. Instead, variable CTCF/cohesin-mediated DNAlooping determines the topological association of transcriptional enhancers with subsets of alternative clustered protocadherin promoters, and these associations drive expression of particular isotypes in individual neurons (Jin and Li 2019; Lefebvre 2017; Mountoufaris et al., 2018). Neuronal self-avoidance requires a functional relationship between allorecognition and chemotactic systems, but this link is not fully understood. The $D$. discoideum allorecognition system shares this relationship with the organism's well-characterized and highly conserved chemotactic system. Insights from $D$. discoideum may thus guide the study of this relationship in other organisms.

Dictyostelium development provides an example of interindividual allorecognition that constrains functional heterogeneity in a eukaryotic, facultatively multicellular amoeba. We will describe this system in depth in the remainder of this review, after discussing several general conclusions from this comparative analysis of cellular allorecognition.

\section{Shared structures and functions in cellular allorecognition systems}

Allorecognition is ubiquitous but it has evolved independently in many different ways in response to different evolutionary pressures. Nevertheless, the different systems share some striking similarities. In terms of structure, often the underlying genetic diversity is expressed in the form of transmembrane proteins that exhibit isoform-specific binding, although the ids locus of $P$. mirabilis is a notable exception. In terms of function, many of the allorecognition systems mediate their effects through modified cell-cell adhesion and/or motility. In more direct comparisons, there are numerous examples of convergent evolution-fruiting body formation in Myxococcus and Dictyostelium, adaptive immunity in both prokaryotes and vertebrates, fusion/rejection responses in Botryllus and Hydractinia, neural self-avoidance mediated by Dscam1 in flies and clustered protocadherins in vertebrates, and multiple forms of plant self-incompatibility systems (Fujii et al., 2016). This frequency of convergent evolution shows the outsized evolutionary advantages that allorecognition systems confer to organisms. It also suggests that allorecognition systems in tractable model organisms and humans may share many basic structures and functions. That these systems underlie such fundamental elements of human health as immunity, cancer, and proper neural function further argues for their translational relevance.

Unfortunately, there are few truly tractable model organisms to study cellular allorecognition. Molecular and genetic characterization of many of the examples provided above is incomplete and often hampered by difficulties developing ready methods for transgenesis and screening. Moreover, in many of these systems, it is difficult to comprehensively link functions to fitness outcomes within appropriate ecological contexts. In contrast, Dictyostelium is readily amenable to genetic manipulation and screening to interrogate its allorecognition system (Kibler et al., 2003; Li et al., 2015). Additionally, our understanding of the ecological context in which allorecognition occurs is relatively well-informed (Tarnita 2017). We will now detail our current understanding of Dictyostelium's allorecognition system and its roles in social evolution and development. We will first introduce $D$. discoideum's developmental 
cheating phenotype because one function of $D$. discoideum's allorecognition system is probably to defend against cheating strains (Ho et al., 2013). Another important feature of the Dictyostelium allorecognition system to establish before we discuss this phenotype is that it does not lead to the exclusive, discrete outcomes of strain coordination or segregation during development. Instead, varying degrees of coordination and segregation are possible, indicating that Dictyostelium allorecognition is a continuous trait (Ostrowski et al., 2008; Gruenheit et al., 2017).

\section{Developmental cheating in Dictyostelium is a model of cellular social evolution}

The first potential report of allorecognition in Dictyostelium came from Leo Buss in 1982. Buss isolated Dictyostelium mucoroides from soil samples and found several chimeric fruiting bodies, i.e. fruiting bodies that contained spores from more than one strain. $\mathrm{He}$ isolated one strain that exhibited normal fruiting body morphology, and a second strain he called "stalkless" that produced balls of spores, but no stalks. He mixed these two strains at varying proportions, developed them, and repeated this process for ten generations. He found that when the stalkless mutant was rare in the starting mix, it could increase to a fixed equilibrium proportion of $\sim 5 \%$ of the spore population within several generations. When the stalkless mutant comprised more than this equilibrium value in the initial mix, it rapidly eliminated the normal-looking cells. Because of these results, Buss called the stalkless strain a somatic cell parasite. He isolated ten additional strains from nearby soil that were not morphologically chimeric, then mixed and developed the stalkless strain at equal proportion with each. In all cases, he found no chimeric fruiting bodies, which implies that the strains possess a property he called a somatic cell compatibility system to defend against somatic cell parasitism (Buss 1982). However, he did not determine whether the strains co-aggregated and then segregated, or simply failed to co-aggregate. Unfortunately, these strains were lost so this important distinction remains unknown. Regardless, although the terms used to describe these phenomena in Dictyostelium have since changed from 'somatic cell incompatibility system' to 'allorecognition system' and from 'somatic cell parasitism' to 'cheating', this single study laid much of the foundation of allorecognition research in Dictyostelium.

The next steps towards establishing Dictyostelium as a model organism for cellular social evolution were published some 18 years later. Strassman et al. generalized and expanded upon Buss's discoveries using the better-studied and more genetically tractable species Dictyostelium discoideum. They used genotyping by PCR of microsatellite loci to demonstrate that fifteen pairwise mixes of naturally isolated $D$. discoideum clones formed chimeric slugs. In half of the chimeric slugs that they tested, one strain contributed more than expected to the prespore population. In terms of the size of this effect, the overrepresented strains' relative contributions to the prespore population were about $10-50 \%$ greater than expected. The authors suggested that competition exists between strains at the level of their relative contributions to the spore population (Strassman et al., 2000).

Ennis et al. provided the first evidence that this developmental cheating phenotype is genetically encoded. The authors performed a genetic screen using restriction enzyme-mediated integration (REMI) mutagenesis, from which they isolated a mutant called
CheaterA ( $c h t A^{-}$, a.k.a. $\left.f b x A^{-}\right)$. This mutant is an obligate cheater in that it preferentially forms spores when developed in chimerae, but does not complete development in a pure population (Ennis et al., 2000). Because this screen yielded only one strain, it was possible that the genetic potential for cheating in Dictyostelium was rather limited. Santorelli et al. refuted that possibility when they expanded the discovery into facultative cheating, in which the cheating strains contribute disproportionately to the spore mass in chimeras, but can also complete clonal development. The authors isolated facultatively cheating strains from another REMI screen and found that they contained mutations in over 100 genes with collectively diverse functions (Santorelli et al., 2009). This study showed that facultative cheating is not limited genetically and that the pathways involved in cheating are quite variable. Khare and Shaulsky demonstrated that the cheating mechanism of one of these REMI mutants called cheaterC (cht $\left.C^{-}\right)$involves a defect in maintaining the prestalk cell fate late in development (Khare and Shaulsky 2010). Santorelli et al. discovered another cheating mechanism that acts through inhibition of the prespore fate in wild-type cells by characterizing a second REMI mutant called cheaterB (chtB-) (Santorelli et al., 2013). However, most of the cheating mechanisms remain unknown.

The Strassman-Queller lab followed up their previous study, addressing a limitation of Buss's work, by systematically testing whether $D$. discoideum forms chimeric slugs in nature. They answered this question by isolating amoebae from very small soil samples. They mixed and developed pairs of clones that cooccurred in the same small soil samples, genotyped them during slug phase, and found that both clones were present in each of the slugs. This finding suggests that in slug phase, chimera formation, and by extension the threat of developmental cheating, is likely common in nature (Fortunato et al., 2003). This result substantiated Buss's original hypothesis that the function of the allorecognition system is to protect potential victims from cheating strains that they encounter frequently in nature.

The Strassman-Queller lab went on to analyze the extent of developmental chimerism in D. discoideum. In 2007, they published results on genotyping of individual fruiting bodies that they isolated from deer dung in nature. They found that $77 \%$ of 88 individual fruiting bodies consisted of a single clone. They demonstrated that such high relatedness can protect potential victims in and of itself in the specific case of cheating by $f b x A^{-}$(Gilbert et al., 2007). This study highlights the importance of two parameters with respect to Dictyostelium allorecognition: the developmental stage and the ecological context in which we assess chimerism and cheating. Through mechanistic studies that we will discuss shortly and ecological studies (Flowers et al., 2010; Douglas et al., 2011), respectively, the field as a whole has begun to clarify the influences of these two parameters. However, the relative prevalence and evolutionary significance of intraspecific interactions in soil as opposed to more contained, nutrient-rich environs like dung, remain unclear.

Ostrowski etal. published another critical piece of evidence for the presence of an allorecognition system in $D$. discoideum (Ostrowski et al., 2008). They grew cells from different strains separately, mixed them in pairs, then developed them under standard laboratory conditions. They found that $D$. discoideum isolates obtained from different locations co-aggregate and then partially segregate. Moreover, the extent of this segregation correlates positively with the genetic distance between pairs; close relatives tend to remain 
co-aggregated whereas genetically distant clones segregate from one another. The genetic distance between the strains was evaluated by microsatellite analysis, as in Strassmann et al., 2000.

\section{The mechanism of Dictyostelium allorecognition}

While Dictyostelium became a model organism for cellular social evolution, researchers concurrently and unwittingly explored an aspect of the biochemistry of the allorecognition system as early as 1976. One of these studies explored the function of the plasma membrane glycoprotein gp150, which was described originally as a cell-cell adhesion protein. It took nearly 25 years to identify that the gene lagC (for loose aggregate C, Dynes et al., 1994) encodes gp150 (Wang et al., 2000), and another 10 years to connect this gene to the allorecognition system (Benabentos et al., 2009; Hirose et al., 2010), at which point it was renamed tgrC1 (tigerC1, abbreviated transmembrane, IPT (Ig-like, plexins, transcription factors), IG (immunoglobulin), E-set (early set; a subset of Ig domains), repeat protein $\mathrm{C} 1$ ) to reflect its protein domain structure and identity as a member of the larger tiger gene family.

\section{Biochemical and developmental characterization of gp150}

The Lerner lab performed the initial characterization of gp150. Geltosky et al. identified this glycoprotein in $D$. discoideum plasma membrane samples and found that its levels increase dramatically between 6 and 18 hours of development. They purified the protein, raised antibodies against it, and demonstrated that these anti-gp150 antibodies inhibit reaggregation of dissociated postaggregative cells (Geltosky et al., 1976). They also used electron microscopy to reveal the cellular localization of gp150, which resides at the plasma membrane and is associated with cell-cell contacts (Geltosky et al., 1980).

Several additional studies from the Lerner and Siu labs further characterized the anti-gp150 antibodies' inhibition of reaggregation in postaggregative cells. Lam et al. demonstrated that prespore cells are more sensitive than prestalk cells to dissociation using anti-gp150 antibodies. They concluded that this phenomenon is due to differential expression of gp150 in these two cell types (Lam et al., 1981). Siu et al. found that treatment with these antibodies also inhibited prestalk/prespore cell sorting into distinct compartments in cells attempting to reaggregate (Siu et al., 1983). Gao et al. discovered that addition of purified gp150 itself to dissociated postaggregative cells also inhibits reaggregation. The authors also found that binding of gp150 to cells is dose-dependent, saturable, and abolished with protease treatment of gp150. These observations suggest that specific binding sites are present on cells, and that intact protein domains are necessary for binding (Gao et al., 1992). The collective results of these studies provided a detailed biochemical characterization of gp150 that continues to guide cell biological and genetic approaches to understand the allorecognition system.

\section{Genetic and developmental characterization of lagC which encodes gp150}

Dynes et al. published the initial genetic characterization of the gene lagC in 1994. The authors isolated the lagC ${ }^{-}$strain from a REMI screen for developmental mutants. They found that lagC mRNA is absent during vegetative growth and 4 hours of development, peaks at 8 hours, and persists through terminal development. Transcriptomic analysis of $D$. discoideum development has validated these results (Rosengarten et al., 2015; Fig. 1B). Consistent with these expression data, the developmental phenotype of the lag $C^{-}$strain includes loose aggregate arrest, in which cells undergo multiple rounds of aggregation and disaggregation. When developed as a pure population, the lag $C^{-}$cells form virtually no spores by 72 hours post-starvation, and demonstrate aberrant or absent expression of cell-type specific markers. Expression of these differentiation markers is partially restored in chimeric development with wild-type cells. However, lag $C^{-}$cells still form very few spores in chimerae. Ectopic overexpression of lagC results in a developmental delay at the tight aggregate stage (Dynes et al., 1994).

Sukumaran et al. followed up on this initial characterization by investigating the morphology of lag $C^{-}$mounds and the epistatic relationship between lag $C$ and $g b f A$. This latter gene is a transcription factor and regulator of post-aggregative and cell-type-specific gene expression (Schnizler et al., 1994). The impetus for this study was the observation that $g b f A^{-}$cells, like lag $C^{-}$cells, arrest at the loose aggregate stage, and feature rounds of aggregation, disaggregation, and reaggregation. The authors found that lag $C^{-}$ mounds do not rotate like wild-type mounds. However, when the authors overexpressed $g b f A$ in this lag $C^{-}$background, the cells formed morphologically normal mounds and completed development (Sukumaran et al., 1998).

Wang et al. performed mass spectrometry of gp150 and identified lagC as the gene that encodes this protein. They showed that gp150 is responsible for EDTA-resistant cell-cell adhesion during aggregation. Because cells that overexpress gp150 during early development do not exhibit this EDTA-resistant cell-cell adhesion, they concluded that gp150 binds heterophilically to another component that is first expressed during aggregation (Wang et al., 2000).

Kibler et al. designed a genetic screen to isolate developmental mutants that sporulate when developed chimerically, but fail to sporulate when developed in a pure population. One of the strains that they identified possessed a null allele of lagC. When they performed pairwise mixing and development of lagC cells with each of the other mutants isolated from the screen, they found that the lag $C^{-}$cells could sporulate with all except for two mutants, which harbored null alleles of the genes lagD or $\operatorname{com} C$. This finding suggested that these three genes are part of one developmental pathway. Accordingly, they investigated the epistatic relationships between the three genes and generated a model in which comC and lag $D$ participate in the transcriptional regulation of lagC (Kibler et al., 2003).

Iranfar etal. provided additional epistatic details about the regulation of lagC expression. They validated that $g b f A^{-}$and lag $C^{-}$cells do not express a post-aggregative gene set, and that $g b f A$ expression is necessary for lag $C$ expression. Additionally, they generated a $g b f A^{-}$, lagC-overexpressing strain, which also did not express the post-aggregative gene set. Based on these observations and the previous finding that lagC-overexpressing cells with intact $g b f A$ exhibit developmental delay at the mound stage (Dynes et al., 1994), they concluded that $g b f A$ and lag $C$ form a feed-forward loop that integrates temporal signaling associated with $g b f A$ expression and cell-cell contact signaling associated with lagC to drive the synchronous expression of post-aggregative genes (Iranfar et al., 2006). 


\section{$\operatorname{tgr} B 1$ and $\operatorname{tgr} C 1$ (a.k.a. lagB1 and lagC1) mediate $D$ discoideum allorecognition}

Benabentos et al. discovered that $\operatorname{lag} C$, which was renamed $\operatorname{lag} C 1$, and a second gene called lagB1, are highly polymorphic and likely under balancing selection in wild isolates (Benabentos et al., 2009). lagB1 is located immediately next to lagC1 in a head-tohead configuration on Chromosome 3 and the two genes share a promoter region. Based on protein structural prediction algorithms, lagB1's protein domains and their structure are somewhat similar to those of lagC1. Specifically, both genes have predicted singlepass transmembrane domains and possess multiple extracellular immunoglobulin-like domains (Fig. 1A). However, the cytoplasmic tail of lagB1 is longer than that of lagC1. That these genes are so tightly linked and polymorphic in and of itself suggested that they may function in allorecognition, as illustrated by convergent evolution of similar loci in other organisms, such as the ids genes in Proteus (Cardarelli et al., 2015), fuhc in Botryllus (Taketa and De Tomaso 2015), and Alr1 and Alr2 in Hydractinia (Nicotra et al., 2009). Benabentos et al. proposed that balancing selection at this locus may have yielded the requisite genetic heterogeneity for kin discrimination to arise. They generated lagB1- cells and showed that, similar to lagC1- cells, the mutant cells exhibited arrested development as loose aggregates. Notably, this arrest in $\operatorname{lagB1} 1^{-}$cells is slightly less penetrant than that of $\operatorname{lagC1^{-}}$, as rare mounds can form small fruiting bodies that contain viable spores. They mixed fluorescently labeled lagB1- or lag $\mathrm{C1}^{-}$cells with wildtype AX4 cells and saw the strains segregate into areas enriched for each strain during mound formation. They excluded the possibility that segregation is a general property of cell-cell adhesion deficiency when they observed no segregation between pairwise mixes of AX4 cells and strains possessing a null allele of $c s a A$ or $\operatorname{cad} A$, which encode separate cell-cell adhesion genes (Noegel et al., 1985; Wong and Siu 1986; Queller et al., 2003; Wong et al., 2002; Siu et al., 2004). Lastly, they found that particular stretches of amino acids in LagB1 and LagC1, near the first extracellular lgfold, predicted strain segregation patterns in the wild strains QS32, QS33, and QS38 (Benabentos et al., 2009), suggesting that the initial observation (Ostrowski et al., 2008) about the relationship

Fig. 1. tgr-mediated allorecognition from DNA to segregation during development. $\operatorname{tgr} \mathrm{B} 1$ and $\operatorname{tgr} \mathrm{C} 1$ reside on Chromosome 3 in a head-tohead configuration, and possess multiple IPT (lg-like, plexins, transcription factors) domains (A). Their developmental mRNA expression patterns are quite similar, likely due to their shared promoter. RPKM: Reads Per Kilobase of transcript, per Million mapped reads, a unit of transcript expression that is normalized by transcript size (Stajdohar et al., 2015) (B). If two fluorescently labeled strains with different tgr allotypes are mixed equally (red and green dots on the left), they segregate beginning around 8 hours of development, as seen in the segregated green and red arms of the rotating mound (Hirose et al., 2010). Importantly, segregation between strains is incomplete as illustrated in the slugs and fruiting body stages, right, in which a few incompatible cells are integrated into the largely homogeneous multicellular structures (Fortunato et al., 2003; Ostrowski et al., 2008; Ho and Shaulsky 2015) (C). The transmembrane TgrB1 receptor (cyan) and TgrC1 ligand (magenta) bind heterophilically between cells (Chen et al., 2013, Chen et al., 2014). They mediate primarily head-to-tail adhesive interactions between TgrC1 at the tail of a leading cell and TgrB1 at the front of a following cell (Fujimori et al., 2019). These interactions result in SCAR complex (purple) recruitment and dendritic actin formation (red) at the front of the following cell (Fujimori 2019) (D). between relatedness and segregation could be fully explained by differences in the LagB1 and LagC1 protein sequences.

Hirose et al. built on these findings to provide evidence that the again renamed $\operatorname{tg} C 1$ and $\operatorname{tg} B 1$ genes are necessary and sufficient to mediate attractive allorecognition between $D$. discoideum strains.

A

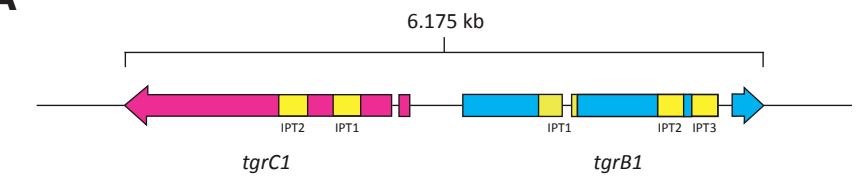

B

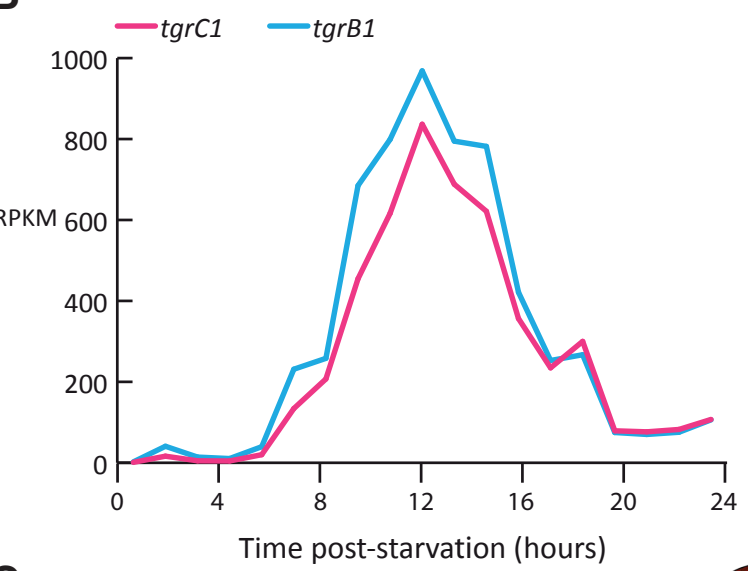

C

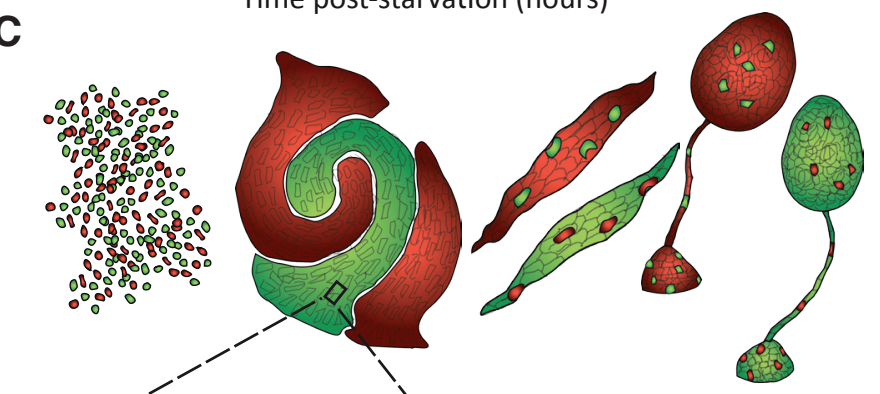

D
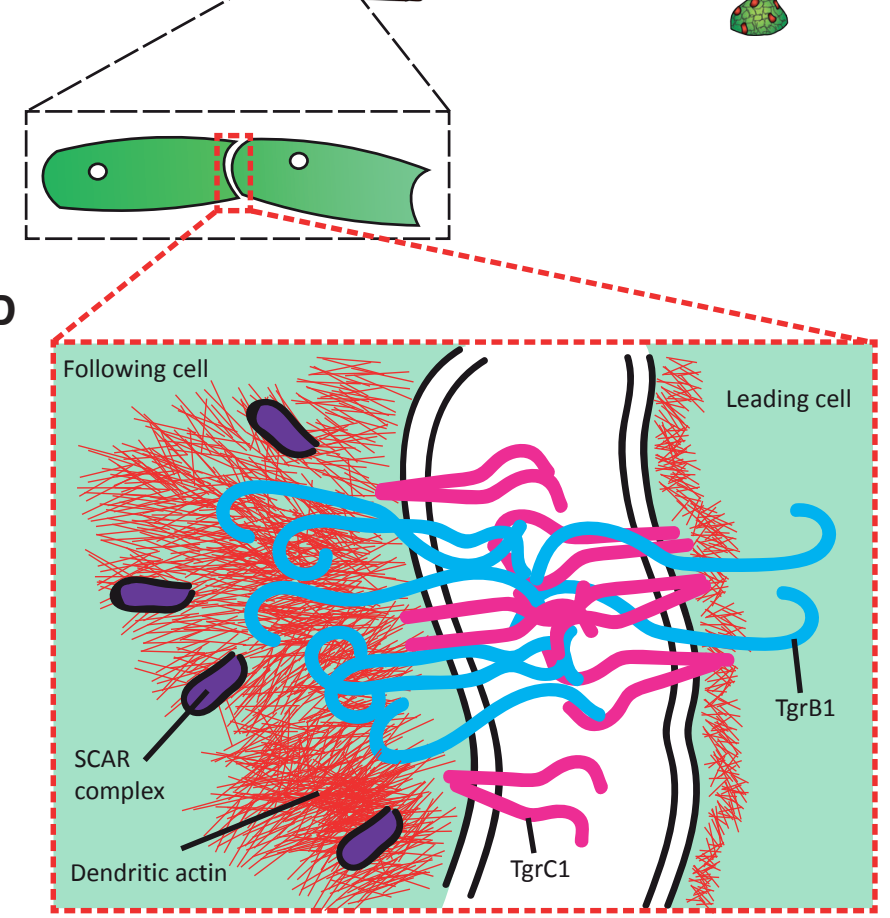
The key strains that allowed the authors to make this conclusion included a tgrB1-; $\operatorname{tgr} C 1^{-}$double-null strain in the AX4 genetic background, and several double gene replacement strains in which the authors replaced the resident $\operatorname{tg} B 1$ and $\operatorname{tg} C 1$ pair with an allelic pair derived from either wild-type AX4 cells or the wild isolates QS4 or QS38. The authors fluorescently labeled each strain, grew the cells separately, and developed them in pairwise mixes. The $\operatorname{tgrB1} 1^{-}$; tgrC $1^{-}$strain demonstrated developmental phenotypes similar to those of $\operatorname{tgrB1} 1^{-}$. Each of the complemented strains exhibited normal development in a pure population, showing that a matching pair of alleles was sufficient for self-compatibility. However, they demonstrated different segregation patterns when mixed equally with AX4 cells (Fig. 1C). The $\operatorname{tgrB} 1^{A X 4} ; \operatorname{tgrC} 1^{A X 4}$ strain could coaggregate with wild-type AX4 cells as expected. However, both $\operatorname{tgrB1} 1^{Q S 4} ; \operatorname{tgrC} 1^{Q S 4}$ and $\operatorname{tgrB} 1^{Q S 38} ; \operatorname{tgrC} 1^{Q S 38}$ strains segregated from $\mathrm{AX} 4$, as well as from each other. In turn, the $\operatorname{tgrB} 1^{Q S 4} ; \operatorname{tgrC} 1^{Q S 4}$ and $\operatorname{tgrB1} 1^{Q S 38}$; $\operatorname{tg} C 1^{Q S 38}$ strains could each co-aggregate and complete development when mixed with the wild strain that possessed matching $\operatorname{tg} B 1$ and $\operatorname{tg} C \mathrm{C} 1$ alleles. These results support the conclusion that $\operatorname{tgr} B 1$ and $\operatorname{tg} C \mathrm{C} 1$ are necessary and sufficient in an exclusive manner for allorecognition. The authors also confirmed that strain segregation is a product of differential adhesion. Lastly, they tested whether allorecognition was attractive or repulsive by generating merodiploid strains that possess two allelic pairs of $\operatorname{tgrB} 1$ and $\operatorname{tg} C \mathrm{C} 1$ genes from separate genetic backgrounds (e.g. $\operatorname{tgr} B 1^{A X}$; $\operatorname{tgrC} 1^{A X 4}$; $\operatorname{tgrB1} 1^{Q S 4} ; \operatorname{tgrC} 1^{Q S 4}$ ) and mixing them with strains with a single matching or non-matching allelic pair. They found that these merodiploid strains could co-aggregate with any strain with which they shared an allelic pair of $\operatorname{tgrB} 1$ and $\operatorname{tgrC} 1$ genes. This finding suggests that allorecognition is attractive rather than repulsive. The merodiploid strains did not co-aggregate with strains that contained an incompatible allelic pair, which suggests that the merodiploid strains are not promiscuous (Hirose et al., 2010).

\section{Mechanistic insights into tgrB1 and $\operatorname{tg} C 1$ functions in the separable processes of allorecognition and development}

In two related studies, the Siu lab explored the biochemical interactions between TgrB1 and TgrC1. In the first study, Chen et al. showed that TgrB1 and TgrC1 bind heterophilically. The authors also demonstrated that addition of anti-TgrB1 antibodies to dissociated postaggregative cells can inhibit reaggregation, like anti-TgrC1 antibodies in previous work (Geltosky et al., 1979). Finally, the authors mapped the sites of interaction between these two proteins to short runs of amino acids in the TIG3 domain of TgrB1 and the TIG2 domain of TgrC1 (Chen et al., 2013). In a later study, the authors further characterized the TgrB1-TgrC1 interaction. Their results suggest a model of interaction in which transmembrane TgrC1 proteins spontaneously dimerize on the membrane of individual cells, i.e. cis-homodimerize. Next, these TgrC1 cis-homodimers bind TgrB1 proteins on an adjacent cell, i.e. trans-interact, which leads to cis-homodimerization of TgrB1 in the adjacent cell. This cis-homodimerization of TgrB1 then induces clustering of TgrB1 and TgrC1 into large adhesion complexes comprised of many trans-interacting, cis-homodimerized TgrB1 and TgrC1 proteins (Chen et al., 2014) (Fig. 1D).

Hirose et al. showed that the allorecognition system imposes a general gating step on developmental progression. They made this discovery by following individual cells in the context of a rotating mound and comparing the behaviors of rare allotype-incompatible individuals to the behaviors of control cells of a different allotype in the same population. Using this setup, the authors demonstrated that these minority, allotype-incompatible cells fail to polarize, to exhibit coordinated movement with the rotating aggregate of majority cells, and to differentiate into prespore and prestalk cell types. They also found that both majority and minority cells' responsiveness to CAMP gradients decreases during the transition from streaming aggregation to mound formation. Lastly, consistent with previous work, they showed that cell-cell contact is required for cell type differentiation because there is a critical cell density below which contact and differentiation do not occur (Kay 1987; Hirose et al., 2015).

Shortly thereafter, Gruenheit et al. demonstrated that the tgr locus, which comprises $\operatorname{tg} B 1$, $\operatorname{tg} C 1$, and their shared promoter region, constitutes a polymorphic green-beard locus (Gruenheit et al., 2017), i.e. the polymorphism of the tgr locus provides the requisite genetic diversity to selectively confer fitness benefits to conspecifics that express the same alleles. Researchers of most other green-beard loci, such as the ids genes of Proteus (Cardarelli et al., 2015) and yeast flo1 (Smukalla et al., 2008), have not demonstrated this aspect of green-beard function, which is likely crucial for controlling cheating (Jansen and Baalen 2006). To our knowledge, the only other known polymorphic green-beard is $\operatorname{tra} A$, which affects cooperative swarming motility in $M$. xanthus (Pathak et al., 2013). However, traA's overall effects on fitness remain somewhat unclear (Wielgoss et al., 2018). To demonstrate that tgr is a polymorphic green-beard, the authors determined the segregation patterns of 20 D. discoideum strains, and found that about $80 \%$ of them exhibit some amount of segregation. They determined that segregation patterns do not correlate with the overall genetic distance between the strains, but do correlate with the allelic similarity of the tgr locus. They expressed and purified from bacteria the TgrB1 and TgrC1 proteins from each of four representative strains, and showed that the strength of binding between TgrB1 and TgrC1 pairs correlates with the degree of segregation. Finally, they demonstrated that forced developmental chimerism imposes a higher fitness cost on pairs of strains that would normally segregate than on pairs that would normally co-aggregate in the form of diminished distances of slug migration (Gruenheit et al., 2017).

Hirose et al. confirmed and extended Gruenheit et al.'s experiments that demonstrated the binding specificity between matching pairs of TgrB1 and TgrC1. To ensure proper glycosylation, the authors expressed in $D$. discoideum the extracellular domains of each protein pair from five additional strains. They mixed them and observed binding exclusively between matching pairs. Additionally, they built on their previous experimental setup (Hirose et al., 2015) to suggest that TgrB1 acts cell-autonomously as a receptor, and TgrC1 acts non-cell-autonomously as TgrB1's ligand. They further supported these arguments by exploring the properties of TgrB1's cytoplasmic tail, dynamic phosphorylation state, and several dominant mutations. Li et al. had previously identified these dominant mutations in a chemical mutagenesis screen for mutations that suppressed the lack of sporulation of a parental strain with non-compatible $\operatorname{tgrB} 1$ and $\operatorname{tgrC} 1$ alleles $\left(\operatorname{tgrB} 1^{A X 4} ; \operatorname{tg} \operatorname{tg}^{Q S 38}\right)$ (Li et al., 2016). Hirose et al. determined that these dominant alleles of $\operatorname{tgrB1}$, which encode amino acid changes in both the cytoplasmic tail and the extracellular domain, contain activating, gain-of-function mutations. Strains that possess one of these $\operatorname{tg} r B 1$ alleles do not require $\operatorname{tg} C 1$ to differentiate and complete development (Hirose 
et al., 2017).

Results from two studies suggest that the dual functions of $\operatorname{tgrC} 1$ in development and allorecognition are separable. In the first, Wang and Shaulsky performed a REMI screen for suppressors of the sporulation defect of $\operatorname{tgr} C 1^{-}$cells, and isolated a mutant called stc $A^{\text {ins }}$ (suppressor of $\operatorname{tgr}\left(1^{-}\right.$), which encodes a putative protein of 557 amino acids that lacks distinctive features. They found that while stc $A^{\text {ins }}$ does modulate sporulation efficiency and thus development of $\operatorname{tgrC} 1^{-}$cells, it does not affect allorecognition (Wang and Shaulsky 2015). In the second study, Li et al. characterized another suppressor of the tgrC1- sporulation defect in the form of a null allele of alg9, which encodes a putative mannosyl transferase involved in $\mathrm{N}$-linked protein glycosylation. This mutation partially complements the sporulation defect of $\operatorname{tg} C 1^{-}$and $\operatorname{tgrB} 1^{A X 4}$; $\operatorname{tgrC} 1^{Q S 38}$ strains, but similarly to stc $A^{\text {ins }}$, does not affect allorecognition (Li et al., 2015).

Fujimori et al. provided evidence that TgrB1 and TgrC1 adhesion complexes interface with the actin cytoskeleton. The authors demonstrated that these adhesion complexes exhibit juxtacrine signaling from TgrC1 located at a leading cell's tail to TgrB1 located at a following cell's front. They showed that this interaction results in SCAR complex recruitment and dendritic actin formation at the front of the following cell. They also documented a difference in the responsiveness of prestalk versus prespore cells to the competing chemotactic input of a cAMP gradient. They demonstrated that prestalk cells tend to orient toward cAMP, while prespore cells tend to orient toward tgr-mediated cell-cell contacts. This observation may account for the sorting of prestalk and prespore cells in the developing mound. While they did not examine juxtacrine signaling interactions in multiple allotypes, these interactions may play a role in allorecognition by influencing segregation between allotypeincompatible strains (Fujimori et al., 2019; Fig. 1D).

\section{The role of allorecognition in $D$. discoideum social evolution}

Ho et al. showed that $D$. discoideum's allorecognition system can protect cooperating strains from cheating strains. The authors accomplished this by determining the fitness costs in terms of spore production between several strains that differed only in their tgr allotypes and their ability to cheat, as conferred by loss of function of $f b x A$. They demonstrated that compared to allotype-compatible strains, allotype-incompatible strains incur decreased fitness costs when they are co-developed with a cheating strain. They also showed that this protection depends on the allotype-incompatible strain's ability to segregate from the cheater. The threat of cheating may thus drive an evolutionary arms race between cheating and allorecognition. That possibility, and the vast genetic potential for cheating, could account for the high degree of polymorphism found in the tgr locus (Ho et al., 2013).

Ho and Shaulsky went on to define a mechanism by which $D$. discoideum utilizes temporal regulation of allorecognition to maximize the benefits and reduce the costs associated with chimerism. The authors found that when rare allotype-incompatible cells are mixed with a majority of cells of a different allotype, these rare cells are initially excluded from aggregates, but can ultimately join slugs and form spores. They also demonstrated that cheating is generally suppressed during later development. These observations suggest that temporal regulation of allorecognition enables strains to avoid some of the costs of chimerism by segregating during late aggregation, a critical period for cell-type differentiation and the threat of cheating. It then enables the benefits of chimerism, such as prolonged slug migration and increased spore dispersal, to accrue by rendering the allorecognition system nonfunctional during later development (Smith et al., 2014; Foster et al., 2002; Ho and Shaulsky 2015). Jack et al. phenomenologically tested and confirmed several of these predictions (Jack et al., 2015).

\section{Prospects in D. discoideum allorecognition research}

\section{Co-evolution of tgrB1 and tgrC1}

TgrB1 and TgrC1 protein sequences can be quite different between different strains, with up to $13 \%$ differences in the amino acid sequences of the extracellular domains. Nevertheless, an organism that carries incompatible $\operatorname{tg} B 1$ and $\operatorname{tg} C 1$ alleles is unable to develop properly. These findings suggest that tgrB1 and $\operatorname{tgrC1}$ must co-evolve somewhat rapidly, potentially even over single organismal lifetimes, as occurs at the genomic level in adaptive immunity (Hille et al., 2018; Rock et al., 2019) and the level of gene expression in neural self-avoidance (Jin and Li 2019). Several potential mechanisms may explain the rapid co-evolution of $\operatorname{tg} B \mathrm{~B} 1$ and $\operatorname{tg} C 1$. First, there is evidence of duplication of the polymorphic $\operatorname{tg} B 1$-tgrC1 locus, but not of the tgrD1-tgrE1 locus, which is similar to the tgrB1-tgrC1 locus but is not polymorphic (Benabentos et al., 2009). Because the allorecognition system is inclusive (Hirose et al., 2010), a duplication event that results in two copies of $\operatorname{tg} B 1$ and tgrC1 might allow cells harboring these multiple alleles to retain cooperative aggregation and differentiation with their parental strain, while relaxing the selective pressure on one of the allelic pairs. Alternatively, incurring a dominant, gain-of-function allele of $\operatorname{tgrB1}$ obviates the need for $\operatorname{tg} C 1$, which could also temporarily relax selective pressure and drive co-evolution. The frequencies and significance of these candidate mechanisms remain to be studied.

\section{Screening and selection methodologies for direct assessment of allorecognition}

To date, the screening methodologies for allorecognition, which include both REMI and chemical mutagenesis to introduce mutations (Wang and Shaulsky 2015, Li et al., 2016), have not directly assessed this process. Instead, they have used as a proxy the suppression of the deficient fruiting body formation in parental $\operatorname{tg} \mathrm{C} 1^{-}$or tgr-mismatched strains. These screens have demonstrated that the segregation of allotype-incompatible strains is separable from the developmental defects that arise from perturbations of the tgrlocus. However, this realization highlights the need for highthroughput methodologies to directly assess strain segregation. Such methodologies may shed light on the extent to which these dual functions are interrelated. These methodologies may also clarify whether the allorecognition pathway represents a step in a dependent sequence that ultimately leads to cell-type differentiation and developmental progression, or if the pathway instead plays a direct role in these processes.

\section{Allorecognition in a broad ecological context}

Several studies have expanded the measures of fitness that allorecognition may affect. In particular, Wolf et al. demonstrated a general fitness tradeoff between spore production and spore viability (Wolf et al., 2015). Additionally, Dubravcic et al. described cells called 'loners' that do not aggregate or participate in the 
formation of multicellular fruiting bodies, but thrive if a food source is reintroduced. These authors showed that strains differ in their allocations to this cell type based on nutritional and genetic factors, as well as the presence of co-aggregating strains (Dubravcic et al., 2014). Several authors have used computational modeling to demonstrate that these observations invalidate the assumption that differences in strains' relative contributions to spores when they are developed as chimeras necessarily arise from cheating. Measuring spore parameters and loners is thus essential for assessing cheating. They suggest that altruism, cheating, and losing can only be defined within this broader appreciation of ecological context (Tarnita et al., 2015; Martinez-Garcia and Tarnita 2016; Tarnita 2017). How the allorecognition system operates within this broader ecological context remains to be studied.

\section{Do other selective pressures influence the evolution of allorecognition?}

Although the available evidence suggests that developmental cheating represents a primary selective force acting on the allorecognition system, other unidentified selective forces may also be at play. One potential additional selective force could derive from findings that some strains of $D$. discoideum carry bacteria throughout multicellular development (Brock et al., 2011), that the identities of the carried bacteria differ between strains (Haselkorn et al., 2018), and that the carried bacteria may both help and harm the amoebae (Brock et al., 2011, DiSalvo et al., 2015). In this context, allorecognition could potentially act as a mechanism for both sharing beneficial bacteria and limiting the transmission of harmful bacteria.

\section{Generality of allorecognition in other dictyostelids}

Several studies of allorecognition in other dictyostelids have been published (Mehdiabadi et al., 2006; Kalla et al., 2011; Sucgang et al., 2011; Sathe et al., 2014; Hayakawa and Inouye 2018), but their collective findings only begin to address the generality of allorecognition among dictyostelids and the extent of conservation of tgr-related mechanisms. Interestingly, Hayakawa and Inouye demonstrated that heterologous expression of the $D$. purpureum homologs of tgrB1 and tgrC1 in D. discoideum is sufficient for cooperative development to occur in mixes of $D$. purpureum and the engineered $D$. discoideum strain. They also found that pairs of $\operatorname{tg} r$ genes exist only within a clade of dictyostelids that produce prestalk cells, which may indicate concurrent evolution of allorecognition and the prestalk cell fate (Hayakawa and Inouye 2018). Curiously, tgrC1 is expressed preferentially in prestalk cells during the slug stage, even though it is expressed rather equally in all cells during late aggregation, whereas tgrB1 does not exhibit strong cell-type preference.

\section{The inclusive nature, pleitropy, and temporal regulation on display in Dictyostelium allorecognition inform the study of other allorecognition systems}

The study of Dictyostelium allorecognition has produced many insights that likely apply to cellular allorecognition systems as a whole. One broadly significant insight is that allorecognition systems may often mediate continuous and inclusive traits. In this context, we define a continuous trait as one that yields a spectrum of interaction between strains (e.g. more or less segregation), as opposed to a binary interaction (e.g. segregation or no segregation). We define an inclusive trait as a beneficial interaction between strains that depends only on the presence of a compatible allotype and is not abolished by the presence of additional incompatible allotypes in any of the strains in question. Characterizations of allorecognition systems tend to focus on easily observable, binary, and exclusive outcomes, but in the case of Dictyostelium, segregation biases strains toward clonal development, rather than constraining them to it. Additionally, the inclusive nature of the system provides potential mechanisms by which it may evolve rapidly in response to selective pressure. The development of experimental setups that allow quantitative determinations of continuous traits related to allorecognition and their genetic underpinnings in other organisms may represent a worthwhile investment in understanding both the immediately relevant traits as well as their evolutionary origins and maintenance.

Another probably often overlooked consideration is that genes associated with allorecognition not only are often pleiotropic in general, but pleiotropic in specific ways that constrain cheating behavior in and of themselves. That loss of function of $\operatorname{tgrB1}$ and/or tgrC1 results in diminished sporulation efficiencies in both clonal and chimeric development provides an example of this phenomenon. Another example of this specific form of pleiotropy that is present in Dictyostelium, but may be independent of allorecognition, is loss of function of $\operatorname{dim} A$. This perturbation causes cells to ignore the signaling molecule DIF-1, which induces prestalk cell differentiation, but concomitantly results in the cells' exclusion from spores (Foster et al., 2004).

Another general insight from $D$. discoideum allorecognition is the importance of temporal regulation of allorecognition systems in maximizing the benefits and minimizing the costs associated with cooperative and competitive behaviors. Several studies document temporal regulation of allorecognition in other organisms, which suggests that it may be widespread (Wilson and Grosberg 2004; Hughes et al., 2004; Downs and Ratnieks 2000). In turn, proper understanding of the significance of temporal regulation requires detailed and comprehensive evaluation of fitness measures, which are sorely lacking in many models of allorecognition.

These insights paint Dictyosteliumallorecognition as an important example of a continuous, inclusive trait whose genetic determinants demonstrate exquisite pleiotropy and temporal regulation, and whose impact on organismal fitness requires careful consideration of ecological context.

\section{Acknowledgements}

We thankDr. Elizabeth Ostrowskifor fruitful discussions, and Dr. Bethany Tiner for assistance using Adobe Illustrator software to generate the figure. This work was supported by grant R35GM118016 from the National Institutes of Health.

\section{References}

ABBASA, LICHTMANAH, PILLAIS(2015). Basic Immunology: Functions and Disorders of the Immune System, Fifth., Ed. R Gruliow. Elsevier, St. Louis.

BENABENTOS R, HIROSE S, SUCGANG R, CURK T, KATOH M, OSTROWSKI EA STRASSMANN JE, QUELLER DC, ZUPAN B, SHAULSKY G, KUSPAA (2009). Polymorphic Members of the lag Gene Family Mediate Kin Discrimination in Dictyostelium. Curr Biol 19: 567-572. https://doi.org/10.1016/j.cub.2009.02.037.

BRETL DJ, KIRBY JR (2016). Molecular Mechanisms of Signaling in Myxococcus xanthus Development. J Mol Biol 428: 3805-3830. https://doi.org/10.1016/j. jmb.2016.07.008. 
BROCK D a, DOUGLAS TE, QUELLER DC, STRASSMANN JE (2011). Primitive agriculture in a social amoeba. Nature 469: 393-396. https://doi.org/10.1038/ nature09668.

BUSS LW (1982). Somatic cell parasitism and the evolution of somatic tissue compatibility. Proc Natl Acad Sci USA 79: 5337-5341.

CARDARELLI L, SAAK C, GIBBS KA (2015). Two Proteins Form a Heteromeric Bacterial Self-Recognition Complex in Which Variable Subdomains Determine Allele-Restricted Binding. MBio 6: e00251-15.

CHEN G, WANG J, XU X, WU X, PIAO R, SIU C-H (2013). TgrC1 mediates cell-cell adhesion by interacting with TgrB1 via mutual IPT/TIG domains during development of Dictyostelium discoideum. Biochem J 452: 259-69.

CHEN G, XU X, WU X, THOMSON A, SIU C-H (2014). Assembly of the TgrB1-TgrC1 cell adhesion complex during Dictyostelium discoideum development. Biochem J 459: 241-249.

DAWKINS R (1976). The Selfish Gene. Oxford University Press.

DISALVO S, HASELKORN TS, BASHIR U, JIMENEZ D, BROCK DA, QUELLER DC, STRASSMANN JE (2015). Burkholderia bacteria infectiously induce the proto- farming symbiosis of Dictyostelium amoebae and food bacteria. Proc Natl Acad Sci USA. 112: E5029-E5037

DOUGLASTE, KRONFORSTMR, QUELLERDC, STRASSMANN JE (2011). Genetic diversity in the social amoeba Dictyostelium discoideum: Population differentiation and cryptic species. Mol Phylogenet Evol 60: 455-462. https://doi.org/10.1016/j. ympev.2011.05.007.

DOWNS SG, RATNIEKS FLW (2000). Adaptive shifts in honey bee (Apis mellifera L.) guarding behavior support predictions of the acceptance threshold model. Behav Ecol 11: 326-333.

DUBRAVCICD, VANBAALEN M, NIZAK C (2014). An evolutionarily significant unicellular strategy in response to starvation stress in Dictyostelium social amoebae. F1000 Res 3: 1-24.

DYNES JL, CLARK AM, SHAULSKY G, KUSPAA, LOOMIS WF, FIRTEL RA (1994). $\mathrm{LagC}$ is required for cell-cell interactions that are essential for cell- type differentiation in Dictyostelium. Genes Dev 8: 948-958.

ENNIS HL, DAO DN, PUKATZKI SU, KESSIN RH, BONNER JT (2000). Dictyostelium amoebae lacking an F-box protein form spores rather than stalk in chimeras with wild type. Proc Natl Acad Sci USA 97: 3292-3297.

FLOWERS JM, LI SI, STATHOS A, SAXER G, OSTROWSKI EA, QUELLER DC STRASSMANN JE, PURUGGANAN MD (2010). Variation, sex, and social cooperation: Molecular population genetics of the social amoeba Dictyostelium discoideum. PLoS Genet 6: e1001013.

FORTUNATO A, STRASSMANN JE, SANTORELLI L, QUELLER DC (2003). Cooccurrence in nature of different clones of the social amoeba, Dictyostelium discoideum. Mol Ecol 12: 1031-1038.

FOSTER KR, FORTUNATO A, STRASSMANN JE, QUELLER DC (2002). The costs and benefits of being a chimera. Proc $R$ Soc London 269: 2357-2362.

FOSTER KR, SHAULSKY G, STRASSMANN JE, QUELLER DC, THOMPSON CRL (2004). Pleiotropy as a mechanism to stabilize cooperation. Nature 431:693-696.

FUJII S, KUBO K, TAKAYAMA S (2016). Non-self- and self-recognition models in plant self-incompatibility. Nat Plants 2: 16130

FUJIMORI T, NAKAJIMA A, SHIMADA N, SAWAI S (2019). Tissue self-organization based on collective cell migration by contact activation of locomotion and chemotaxis. Proc Natl Acad Sci USA 116: 4291-4296.

GAO EN, SHIER P, SIU C-H (1992). Purification and Partial Characterization of a Cell Adhesion Molecule (gp150) involved in Postaggregation Stage Cell-Cell Binding in Dictyostelium discoideum. J Biol Chem 267: 9409-9415.

GELTOSKY JE, BIRDWELL CR, WESEMAN J, LERNERS RA (1980). A Glycoprotein Involved in Aggregation of D. discoideum Is Distributed on the Cell Surface in a Nonrandom Fashion Favoring Cell Junctions. Cell 21: 339-345

GELTOSKYJE, SIU C-H, LERNERSRA(1976). Glycoproteins of the plasma membrane of Dictyostelium discoideum during development. Cell 8: 391-396.

GELTOSKY JE, WESEMAN J, BAKKEA, LERNERS RA (1979). Identification of a cell surface glycoprotein involved in cell aggregation in D. discoideum. Cell18:391-398.

GILBERT OM, FOSTER KR, MEHDIABADI NJ, STRASSMANN JE, QUELLER DC (2007). High relatedness maintains multicellular cooperation in a social amoeba by controlling cheater mutants. Proc Natl Acad Sci USA 104: 8913-8917.

GRUENHEIT N, PARKINSON K, STEWART B, HOWIE JA, WOLF JB, THOMPSON
CRL (2017). A polychromatic "greenbeard" locus determines patterns of cooperation in a social amoeba. Nat Commun 8: 14171 doi: 10.1038/ncomms14171.

HASELKORN TS, BROCK DA, DISALVO S, MILLER JW, BASHIR U, QUELLER DC, STRASSMANN JE (2019). The specificity of Burkholderia symbionts in the social amoeba farming symbiosis : Prevalence, species, genetic and phenotypic diversity. Mol Ecol: 847-862.

HATTORI D, CHEN Y, MATTHEWS BJ, SALWINSKI L, SABATTI C, GRUEBER WB, ZIPURSKY SL (2009). Robust discrimination between self and non-self neurites requires thousands of Dscam1 isoforms. Nature 461: 644-648. https:// doi.org/10.1038/nature08431.

HATTORI D, DEMIR E, KIM HW, VIRAGH E, ZIPURSKY SL, DICKSON BJ (2007). Dscam diversity is essential for neuronal wiring and self-recognition. Nature 449: 223-228.

HAYAKAWA IS, INOUYE K (2018). Species recognition in social amoebae. J Biosci 43: 1025-1036.

HILLE F, RICHTER H, WONG SP, BRATOVIC M, RESSEL S, CHARPENTIER E (2018). Review The Biology of CRISPR-Cas : Backward and Forward. Cell 172: 1239-1259.

HIROSES, BENABENTOSR, HOH, KUSPAA, SHAULSKYG (2010). Self-Recognition in SocialAmoebae Is Mediated by Allelic Pairs of Tiger Genes. Science 333:467-470.

HIROSE S, CHEN G, KUSPA A, SHAULSKY G (2017). The polymorphic proteins TgrB1 and TgrC1 function as a ligand-receptor pair in Dictyostelium allorecognition. J Cell Sci 130: 4002-4012.

HIROSE S, SANTHANAM B, KATOH-KUROSAWA M, SHAULSKY G, KUSPA A (2015). Allorecognition, via TgrB1 and TgrC1, mediates the transition from unicellularity to multicellularity in the social amoebae Dictyostelium discoideum. Development 142: 3561-3570.

HO HI, HIROSE S, KUSPA A, SHAULSKY G (2013). Kin recognition protects cooperators against cheaters. Curr Biol 23: 1590-1595. https://doi.org/10.1016/j. cub.2013.06.049.

HO HI, SHAULSKY G (2015). Temporal regulation of kin recognition maintains recognition-cue diversity and suppresses cheating. Nat Commun 6: 7144. https:// doi.org/10.1038/ncomms8144.

HUGHES RN, MANRIQUEZ PH, MORLEY S, CRAIG SF, BISHOP JDD (2004). Kin or self-recognition? Colonial fusibility of the bryozoan Celleporella hyalina. Evol Dev 6: 431-437.

IRANFAR N, FULLER D, LOOMIS WF (2006). Transcriptional regulation of postaggregation genes in Dictyostelium by a feed-forward loop involving GBF and LagC. Dev Biol 290: 460-469

JACK CN, BUTTERY N, ADU-OPPONG B, POWERS M, THOMPSON CRL, QUELLER DC, STRASSMANN JE (2015). Migration in the social stage of Dictyostelium discoideum amoebae impacts competition. PeerJ 3: e1352.

JANSEN VAA, BAALEN M Van (2006). Altruism through beard chromodynamics. Nature 440: 663-666.

JIN Y, LI H (2018). Revisiting Dscam diversity: lessons from clustered protocadherins Cell Mol Life Sci. 76: 667-680. doi: 10.1007/s00018-018-2951-4

JOHNSON LA, JUNE CH (2017). Driving gene-engineered T cell immunotherapy of cancer. Cell Res 27: 38-58. https://doi.org/10.1038/cr.2016.154.

KALLASE, QUELLERDC, LASAGNIA, STRASSMANN JE (2011). Kin discrimination and possible cryptic species in the social amoeba Polysphondylium violaceum. BMC Evol Biol 11: 1-11.

KARADGE UB, GOSTO M, NICOTRA ML (2015). Allorecognition proteins in an invertebrate exhibit homophilic interactions. Curr Biol 25: 2845-2850. https://doi. org/10.1016/j.cub.2015.09.030.

KAY RR (1987). Cell Differentiation in Monolayers and the Investigation of Slime Mold Morphogens. Methods Cell Biol 28: 433-448.

KHARE A, SHAULSKY G (2010). Cheating by exploitation of developmental prestalk patterning in Dictyostelium discoideum. PLoS Genet 6: e1000854.

KIBLER K, SVETZ J, NGUYEN TL, SHAW C, SHAULSKY G (2003). A cell-adhesion pathway regulates intercellular communication during Dictyostelium development. Dev Biol 264: 506-521.

LAM TY, PICKERING G, GELTOSKY J, SIU CH (1981). Differentiation Differential Cell Cohesiveness Expressed by Prespore and Prestalk Cells of Dictyostelium discoideum. Differentiation 20: 22-28. https://doi.org/10.1111/j.1432-0436.1981. tb01151.x. 
LEFEBVRE JL (2017). Neuronal territory formation by the atypical cadherins and clustered protocadherins. Semin Cell Dev Biol69: 111-121. https://doi.org/10.1016/j. semcdb.2017.07.040.

LIC-LF, CHEN G, WEBBAN, SHAULSKYG (2015). Altered N-glycosylation modulates TgrB1-and TgrC1-mediated development but not allorecognition in Dictyostelium. J Cell Sci 128: 3990-3996.

LI C-LF, SANTHANAM B, NICOLE WEBB A, ZUPAN B, SHAULSKY G (2016). Gene discovery by chemical mutagenesis and whole-genome sequencing in Dictyostelium. Genome Res 26: 1-9.

MARINO J, PASTER J, BENICHOU G (2016). Allorecognition by T Lymphocytes and Allograft Rejection. Front Immunol 7:582 doi: 10.3389/fimmu.2016.00582.

MARTINEZ-GARCIA R, TARNITA CE (2016). Lack of Ecological and Life History Context Can Create the Illusion of Social Interactions in Dictyostelium discoideum. PLoS Comput Biol 12: e1005246.

MEHDIABADI NJ, JACK CN, FARNHAM TT, PLATT TG, KALLA SE, SHAULSKY G, QUELLER DC, STRASSMANN JE (2006). Kin preference in a social microbe. Nature 442: 881-882.

MEYERD, AGUIAR VRC, BITARELLOD, BRANDT DYC, NUNESK(2018). Agenomic perspective on HLA evolution. Immunogenetics 70: 5-27.

MOUNTOUFARIS G, CANZIO D, NWAKEZE CL, CHEN W V., MANIATIS T (2018). Writing, Reading, and Translating the Clustered Protocadherin Cell Surface Recognition Code for Neural Circuit Assembly. Annu Rev Cell Dev Biol 34: 471-493.

NEVES G, ZUCKER J, DALY M, CHESS A (2004). Stochastic yet biased expression of multiple Dscam splice variants by individual cells. Nat Genet 36: 240-246.

NICOTRA ML, POWELL AE, ROSENGARTEN RD, MORENO MA, GRIMWOOD J, LAKKIS FG, DELLAPORTA SL, BUSS LW (2009). A Hypervariable Invertebrate Allodeterminant. Curr Biol 19: 583-589.

NOEGEL A, HARLOFF C, HIRTH P, MERKL R, MODERSITZKI M, STADLER J, WEINHART U, WESTPHAL M, GERISCH G (1985). Probing an adhesion mutant of Dictyostelium discoideum with cDNA clones and monoclonal antibodies indicates a specific defect in the contact site A glycoprotein. EMBO J 4: 3805-3810.

OSTROWSKI EA, KATOH M, SHAULSKY G, QUELLER DC, STRASSMANN JE (2008). Kin discrimination increases with genetic distance in a social amoeba. PLOS Biol 6: 2376-2382.

PATHAK DT, WEI X, DEY A, WALLD (2013). Molecular Recognition by a Polymorphic Cell Surface Receptor Governs Cooperative Behaviors in Bacteria. PLoS Genet 9(11): e1003891.

QUELLER DC, PONTE E, BOZZARO S, STRASSMANN JE (2003). Single-Gene Greenbeard Effects in the Social Amoeba Dictyostelium discoideum. Science (80-) 299: 105-107.

REEVES E, JAMES E (2016). Antigen processing and immune regulation in the response to tumours. Immunology 150: 16-24.

ROCK KL, REITS E, NEEFJES J (2019). Present Yourself! By MHC Class I and MHC Class II Molecules. Trends Immuno/37: 724-737. https://doi.org/10.1016/j. it.2016.08.010.

ROSENGARTENRD, SANTHANAMB, FULLERD, KATOH-KURASAWAM, LOOMIS WF (2015). Leaps and lulls in the developmental transcriptome of Dictyostelium discoideum. BMC Genomics 16: 1-16.

SAAK CC, GIBBS KA (2016). The Self-Identity Protein IdsD Is Communicated between Cells in Swarming Proteus mirabilis Colonies. J Bacteriol 198: 3278-3286.

SANTORELLI LA, KUSPAA, SHAULSKY G, QUELLERDC, STRASSMANN JE (2013). A new social gene in Dictyostelium discoideum, chtB. BMC Evol Biol 13: 1-9.

SANTORELLI L a, THOMPSON CRL, VILLEGAS E, SVETZ J, DINH C, PARIKH A, SUCGANG R, KUSPA A, STRASSMANN JE, QUELLER DC, SHAULSKY G (2008). Facultative cheater mutants reveal the genetic complexity of cooperation in social amoebae. Nature 451: 1107-1110.

SATHE S, KHETAN N, NANJUNDIAH V (2014). Interspecies and intraspecies interactions in social amoebae. J Evol Biol 27: 349-362.

SCHNITZLER GR, FISCHER WH, FIRTEL RA (1994). Cloning and characterization of the G-box binding factor, an essential component of the developmental switch between early and late development in Dictyostelium. Genes Dev 8: 502-514.

SCHUREN ABC, COSTAAI, WIERTZEJHJ (2016). Recent advances in viral evasion of the MHC Class I processing pathway. Curr Opin Immunol 40: 43-50. https:// doi.org/10.1016/j.coi.2016.02.007.

SEED KD (2015). Battling Phages: How Bacteria Defend against Viral Attack. PLoS
Pathog 11: e1004847

SIDDLE H V, KREISS A, TOVAR C, KIT C, CHENG Y, BELOV K, SWIFT K, PEARSE A-M, HAMEDE R, JONES ME, SKJODT K, WOODS GM, KAUFMAN J (2013). Reversible epigenetic down-regulation of $\mathrm{MHC}$ molecules by devil facial tumour disease illustrates immune escape by a contagious cancer. Proc Natl Acad Sc USA 110: 5103-5108.

SIU CH, HARRIS TJC, WANG J, WONG E (2004). Regulation of cell-cell adhesion during Dictyostelium development. Semin Cell Dev Biol 15: 633-641.

SIU C-H, ROCHES B des, LAM TY (1983). Involvement of a cell-surface glycoprotein in the cell-sorting process of Dictyostelium discoideum. Proc Natl Acad Sci USA 80: 6596-6600.

SMITH J, QUELLER DC, STRASSMANN JE (2014). Fruiting bodies of the social amoeba Dictyostelium discoideum increase spore transport by Drosophila. BMC Evol Biol 14(105): 1-5

SMUKALLA S, CALDARA M, POCHET N, BEAUVAIS A, GUADAGNINI S, YAN C, VINCES MD, JANSEN A, PREVOST MC, LATGÉ JP, FINK GR, FOSTER KR, VERSTREPEN KJ (2008). FLO1 Is a Variable Green Beard Gene that Drives Biofilm-like Cooperation in Budding Yeast. Cell 135: 726-737.

STAJDOHAR M, ROSENGARTEN RD, ZUPANB, KOKOSAR J, JERAN L, BLENKUS D, SHAULSKY G (2017). dictyExpress : a web-based platform for sequence data management and analytics in Dictyostelium and beyond. BMC Bioinformatics 18: $1-10$.

STRASSMANN JE, ZHU Y, QUELLER DC (2000). Altruism and social cheating in the social amoeba Dictyostelium discoideum. Nature 408: 965-967.

STRASSMANN JE, GILBERT OM, QUELLER DC (2011). Kin Discrimination and Cooperation in Microbes. Annu Rev Microbiol 65: 349-367.

SUCGANG R, KUOA, TIANX, SALERNOW, PARIKHA, FEASLEYCL, DALINE, TUH, HUANG E, BARRYK, et al., (2011). Comparative genomics of the social amoebae Dictyostelium discoideum and Dictyostelium purpureum. Genome Biol 12: R20.

SUKUMARAN S, BROWN JM, FIRTEL RA, MCNALLY JG (1998). lagC-Null and gbf-Null Cells Define Key Steps in the Morphogenesis of Dictyostelium Mounds. Dev Biol 200: 16-26.

TAKABA H, TAKAYANAGI H (2017). The Mechanisms of T Cell Selection in the Thymus. Trends Immunol 38: 805-816. https://doi.org/10.1016/j.it.2017.07.010.

TAKETA DA, DE TOMASO AW (2015). Botryllus schlosseri allorecognition: Tackling the enigma. Dev Comp Immunol 48: 254-265. https://doi.org/10.1016/j. dci.2014.03.014

TARNITA CE (2017). The ecology and evolution of social behavior in microbes. $J$ Exp Biol 220: 18-24.

TARNITA CE, WASHBURNE A, MARTINEZ-GARCIA R, SGRO AE, LEVIN SA (2015). Fitness tradeoffs between spores and nonaggregating cells can explain the coexistence of diverse genotypes in cellular slime molds. Proc Natl Acad Sci USA 112: 2776-2781.

WANG J, HOU L, AWREY D, LOOMIS WF, FIRTELRA, SIU CH (2000). The membrane glycoprotein gp150 is encoded by the lagC gene and mediates cell-cell adhesion by heterophilic binding during Dictyostelium development. Dev Biol227: 734-745.

WANG Y, SHAULSKY G (2015). TgrC1 has distinct functions in Dictyostelium development and allorecognition. PLoS One 10: e0124270.

WIELGOSS S, FIEGNA F, RENDUELES O, YU Y-TN, VELICER GJ (2018). Kin discrimination and outer membrane exchange in Myxococcus xanthus: Acomparative analysis among natural isolates. Mol ECOl 27: 3146-3158.

WILSON ACC, GROSBERG RK (2004). Ontogenetic shifts in fusion-rejection thresholds in a colonial marine hydrozoan, Hydractinia symbiolongicarpus. Behav Eco Sociobiol 57: 40-49.

WOLF JB, HOWIE JA, PARKINSON K, GRUENHEITN, MELOD, ROZEND, THOMP SON CRL (2015). Fitness Trade-offs Result in the Illusion of Social Success. Curr Biol 25: 1-5. https://doi.org/10.1016/j.cub.2015.02.061.

WONG E, YANG C, WANG J, FULLER D, LOOMIS WF, SIU C (2002). Disruption of the gene encoding the cell adhesion molecule DdCAD-1 leads to aberrant cell sorting and cell-type proportioning during Dictyostelium development. Development 129: 3839-3850.

WONG LM, SIU CH (1986). Cloning of cDNA for the contact site A glycoprotein of Dictyostelium discoideum. Proc Natl Acad Sci USA 83: 4248-4252.

YAMAZAKI K, BEAUCHAMP GK (2007). Genetic Basis for MHC-Dependent Mate Choice. Adv Genet 59: 129-145. 


\section{Further Related Reading, published previously in the Int. J. Dev. Biol.}

YelA, a putative Dictyostelium translational regulator, acts as antagonist of DIF-1 signaling to control cell-type proportioning Yoko Yamada, Chris Sugden and Jeffrey G. Williams

Int. J. Dev. Biol. (2017) 61: 35-42

https://doi.org/10.1387/ijdb.160160yy

An orthologue of the Myelin-gene Regulatory Transcription Factor regulates Dictyostelium prestalk differentiation

Hiroshi Senoo, Hong-Yu Wang, Tsuyoshi Araki, Jeffrey G. Williams and Masashi Fukuzawa Int. J. Dev. Biol. (2012) 56: 325-334

https://doi.org/10.1387/ijdb.120030jw

Dictyostelium discoideum Sir2D modulates cell-type specific gene expression and is involved in autophagy

Rakhee Lohia, Punita Jain, Mukul Jain, Pradeep Kumar Burma, Anju Shrivastava and Shweta Saran

Int. J. Dev. Biol. (2017) 61: 95-104

https://doi.org/10.1387/ijdb.160038ss

Bimodal distribution of motility and cell fate in Dictyostelium discoideum

Pavana Goury-Sistla, Vidyanand Nanjundiah and Gopal Pande

Int. J. Dev. Biol. (2012) 56: 263-272

https://doi.org/10.1387/ijdb.113384ps

Development at the edge of multi-cellularity: Dictyostelium discoideum.

R R Kay

Int. J. Dev. Biol. (2000) 44: 35-38

http://www.intjdevbiol.com/web/paper/10761844
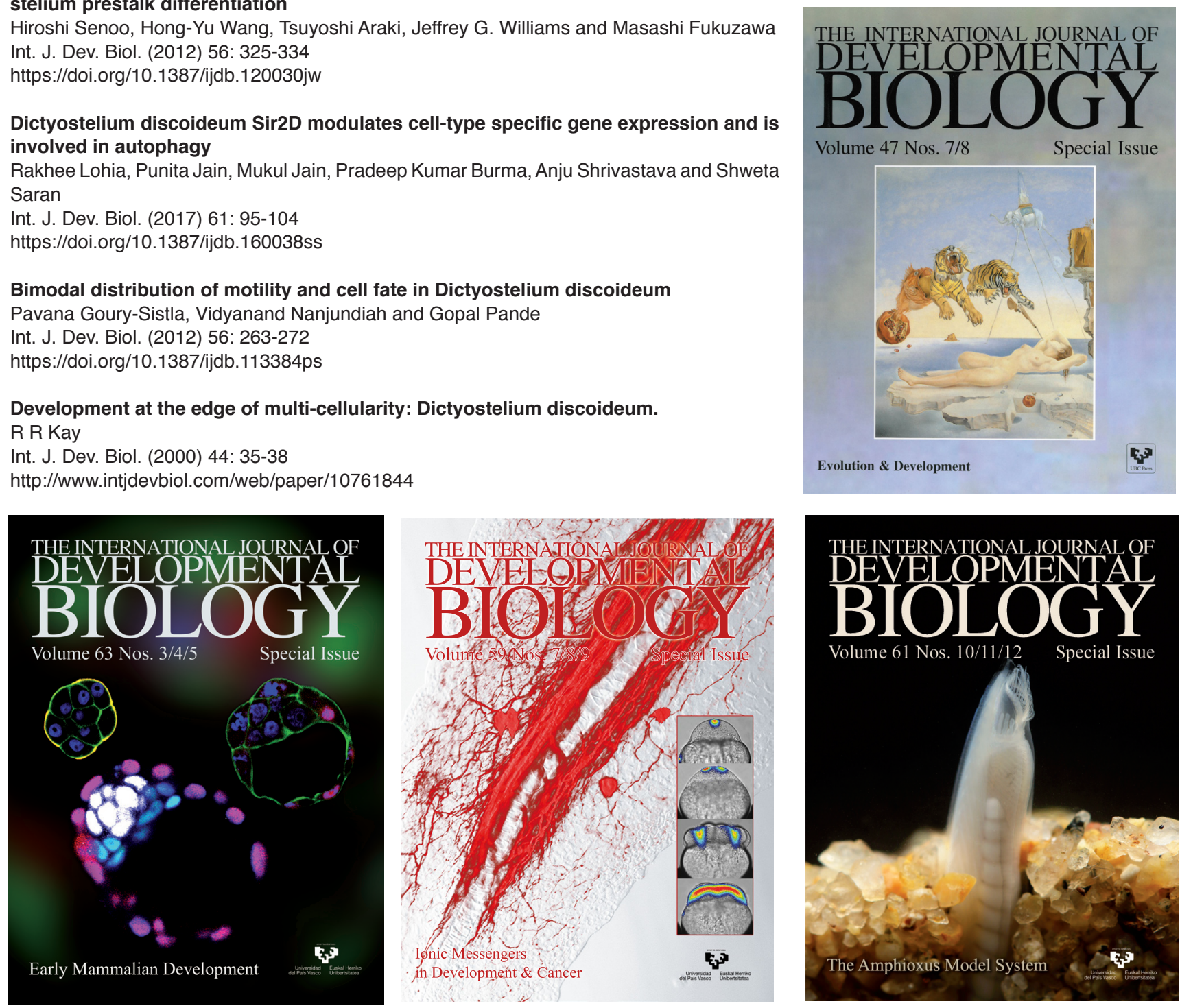